\title{
A case scenario study for the assessment of physician's behavior in the management of COPD: the WHY study
}

This article was published in the following Dove Press journal: International Journal of COPD

\author{
Oguz Kilinc' \\ Aylin Konya ${ }^{2}$ \\ Metin Akgun ${ }^{3}$ \\ Esra Uzaslan ${ }^{4}$ \\ Abdullah Sayiner ${ }^{5}$ \\ 'Department of Chest Diseases, \\ Dokuz Eylül University Medical \\ Faculty, Izmir, Turkey; ${ }^{2}$ Department \\ of Medical Management, Novartis \\ Pharmaceuticals, Istanbul, Turkey; \\ ${ }^{3}$ Department of Chest Diseases, \\ Atatürk University Medical Faculty, \\ Erzurum, Turkey; ${ }^{4}$ Department of \\ Chest Diseases, Uludağ University \\ Medical Faculty, Bursa, Turkey; \\ ${ }^{5}$ Department of Chest Diseases, \\ Ege University Medical Faculty, \\ Izmir, Turkey
}

Purpose: COPD diagnosis is mainly based on clinical judgment of physicians. Physicians do not also refer to COPD guidelines in their daily practice. This study aimed to assess attitudes of physicians regarding COPD diagnosis, treatment, and follow-up and to identify the factors influencing physicians' decisions in clinical practice.

Patients and methods: Fifty physicians were selected from 12 EuroStat NUTS 2 regions and asked to assess seven fictitious case scenarios. The following five scenarios described patients with COPD: Case Global Initiative for Chronic Obstructive Lung Disease (GOLD) A-smoker and Case GOLD A-nonsmoker were previously undiagnosed patients presenting with dyspnea, Case GOLD D-smoker and GOLD B-exsmoker were COPD patients presenting with exacerbation, Case GOLD B-smoker was a previously diagnosed COPD patient with dyspnea in stable phase, Case asthma-COPD overlap syndrome, and Case obesity hypoventilation syndrome. Patients' history, physical examination findings, pulmonary function tests, and X-ray images were prepared before the study by an experts' committee and provided to the physicians upon their request, until they reached a final decision. The physicians completed a questionnaire including information about their clinical practices and institutions.

Results: According to the GOLD 2015 recommendations, of the physicians, 44\% performed guideline-concordant diagnosis in the first five scenarios, who were all COPD patients, and $6 \%$ performed guideline-concordant diagnosis in all cases. There was a negative correlation between high workload and making a guideline-concordant diagnosis $(P=0.038$, rho $=-0.417)$. Even when the physicians made a guideline-concordant diagnosis of COPD, only a minority $(10 \%-22 \%)$ used the GOLD classification. Logistic regression analysis revealed that working in a tertiary health care center was a significant factor in favor of establishing a guidelineconcordant diagnosis of COPD $(P=0.029, \mathrm{OR}=6.139$ [95\% CI: $1.20-31.32])$.

Conclusion: Management of COPD patients in Turkey does not generally follow the GOLD criteria but is rather based on physicians' clinical experience. Heavy workload appears to adversely affect the correctness of clinical decisions.

Keywords: COPD, practice pattern, real-life scenario, physician decision, GOLD criteria

\section{Introduction}

COPD is a global public health concern that causes significant morbidity and mortality with an increasing trend. ${ }^{1}$ In 2011, age and gender standardized national prevalence rates of COPD in Turkey were reported to be $5.3 \%$ and $4.0 \%$, respectively, when the disease was diagnosed based on pulmonary function tests (PFTs) and physicians' assessments. $^{2}$ Owing to the discordance between these two rates, a practice pattern study (ALPHABET) was conducted to examine how COPD patients were evaluated
Department of Chest Diseases, Dokuz Eylül University Medical Faculty, İnciraltı Mahallesi, Mithatpașa Caddesi No 1606, 35340 Narlıdere, Balçova, Izmir, Turkey Tel +905359694099

Email oguz.kilinc@deu.edu.tr (c) (7) (c) 2018 Kilinc et al. This work is published and licensed by Dove Medical Press Limited. The full terms of this license are available at https://www.dovepress.com/terms.php cc. hereby accept the Terms. Non-commercial uses of the work are permitted without any further permission from Dove Medical Press Limited, provided the work is properly attributed. For permission for commercial use of this work, please see paragraphs 4.2 and 5 of our Terms (https://www.dovepress.com/terms.php). 
in real life. ${ }^{3}$ The ALPHABET study showed that the ratio of forced expiratory volume in 1 second $\left(\mathrm{FEV}_{1}\right)$ to forced vital capacity was $\geq 70 \%$ in $40 \%$ of the patients previously diagnosed with COPD. Besides, the most common treatment regimen, which was used in $62 \%$ of COPD patients, was triple therapy, ie, the combination of long-acting $\beta 2$ agonists (LABA), inhaled corticosteroids (ICS), and longacting muscarinic antagonists (LAMA). ${ }^{3}$ The same study also showed that physicians' compliance to multidimensional assessment approach, as outlined by the Global Initiative for Chronic Obstructive Lung Disease (GOLD) strategy, was relatively low. In a study from the United States including the records of 450 patients with COPD confirmed by spirometry, it was demonstrated that $43.6 \%$ of the patients were not receiving guideline-based treatment and, of these patients, who were all GOLD stage I, 7.6\% were overtreated. ${ }^{4}$ Moreover, another study from the United States reported that $23.1 \%$ of the COPD patients received a treatment that was not consistent with any of the guidelines' recommendations irrespective of disease severity. ${ }^{5}$ Based on these findings, the current study, which was called "WHY Study", was designed to evaluate attitudes of physicians regarding COPD diagnosis, treatment, and follow-up and to identify the factors influencing physicians' decisions in clinical practice.

\section{Patients and methods}

The present study was designed as a practice pattern survey and conducted from April to July 2015. The study was approved by the ethics committee of Dokuz Eylul University Medical Faculty (date: December 18, 2014; no: 2014/37-02). Written and signed informed consents of the physicians to participate in the study were obtained.

No actual patients were enrolled; however, fictitious case scenarios were used to represent patients with COPD. For each case scenario, demographic features, medical histories, physical examination findings, and medical and screening test results were prepared prior to the study.

Fifty physicians who were working in different tertiary (university hospitals or training and research hospitals) and secondary (state hospital or private hospital) health care centers and willing to participate in the study were included. The centers were selected from 12 provinces (Adana, Ankara, Samsun, Balikesir, Trabzon, Bursa, Izmir, Gaziantep, Istanbul, Erzurum, Kayseri, and Van) according to the European Community Regional Policies Level 2 Statistical Regional Units (EuroStat NUTS 2 regions) to provide a representative sample of Turkey. In Turkey, the tertiary health care centers are also academic centers, which have residency programs and train pulmonology fellows. These consist of university hospitals and a total of four research and training hospitals for pulmonary diseases. The secondary health care centers are state hospitals, which only provide patient care.

\section{Case scenarios}

All scenarios and related documents were produced by the steering committee of the study based on the recommendations of the GOLD document and the Turkish Thoracic Society's COPD guideline, similar to the methodology used by Fukuhara et al. ${ }^{6}$ The members of the committee were physicians with a special interest in COPD, working as consultants in academic institutions. The participants were expected to order the correct diagnostic tests, to evaluate the quality of the spirometry, to use the right parameters (level of $\mathrm{FEV}_{1}$, symptom score, and history of exacerbations) for classifying the patient, and to choose a treatment regimen that was concordant with the guidelines.

The following five scenarios described patients with COPD: Case GOLD A-smoker and Case GOLD A-nonsmoker were previously undiagnosed patients presenting with dyspnea, Case GOLD D-smoker and GOLD B-exsmoker were COPD patients presenting with exacerbation, Case GOLD B-smoker was a previously diagnosed COPD patient with dyspnea in stable phase. The remaining two cases, which are sometimes misdiagnosed and/or treated as COPD, were Case asthma-COPD overlap syndrome (ACOS) and Case obesity hypoventilation syndrome (OHS). Each case scenario included the results of medical and screening tests that are usually used in clinical practice for diagnosing COPD patients. Detailed description of the case scenarios is presented in Table S1.

The same PFT result sheet was used for all COPD cases. However, Case ACOS and Case OHS had distinctive PFT result sheets. In some cases, some tricky PFT results, compatible with a noncooperative patient, were first given to the physicians, expecting them to request an acceptable PFT result sheet. Similarly, chest X-rays of poor technical quality were also given. Thus, a patient dossier was prepared for each case scenario.

During face-to-face interviews, physicians examined the history and laboratory and radiographic findings and requested additional tests, whenever they felt a need, until they reached a decision for each of the seven case scenarios (Table 1). In these interviews, the GOLD classification of the cases was not asked to the physicians; however, they were evaluated by checking whether they asked for the correct clinical parameters to classify the patients, namely symptom scores, history of exacerbations, and $\mathrm{FEV}_{1}$ levels. 


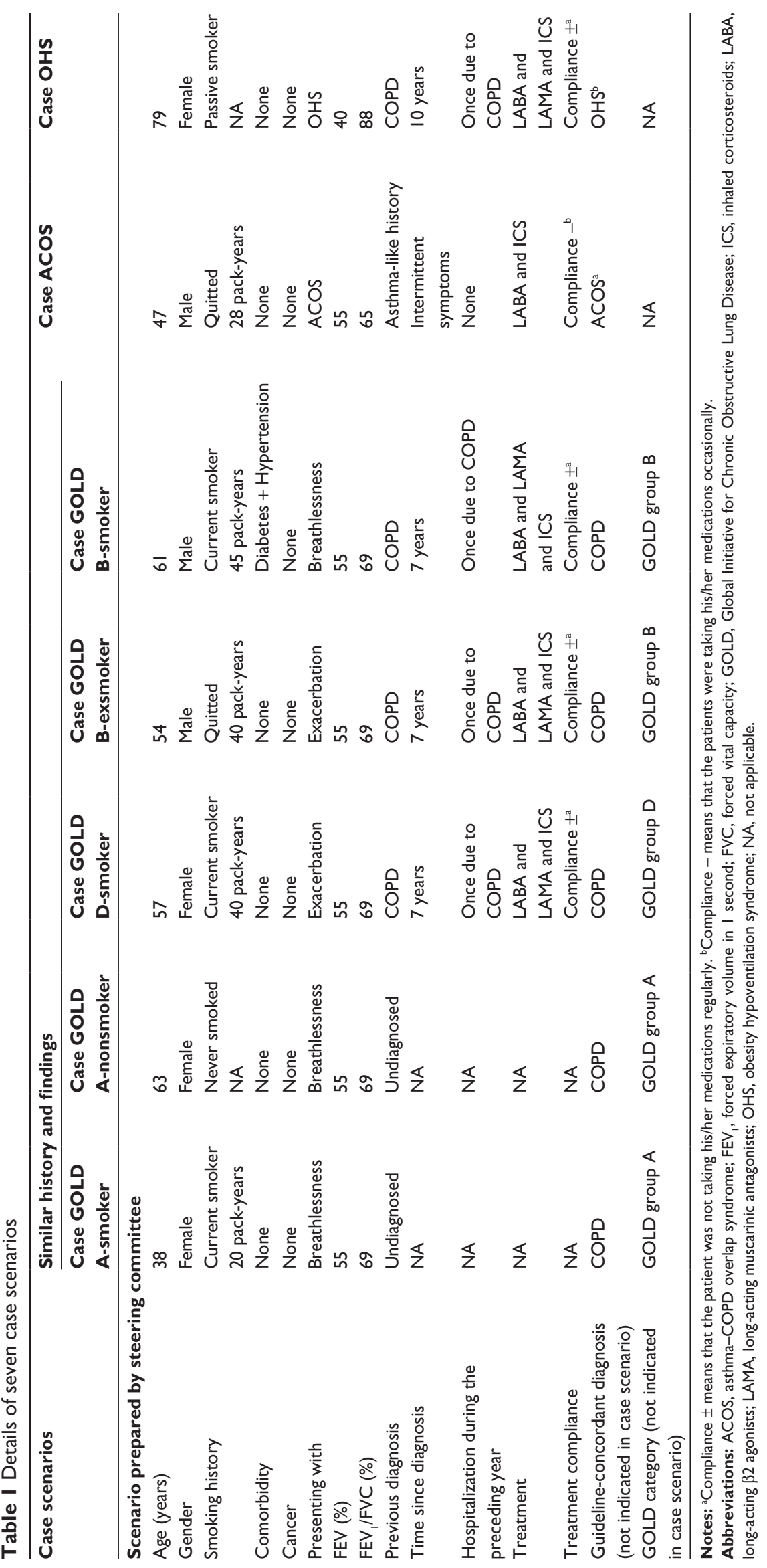


The tests requested by the physicians were recorded. There was no limitation in time for the physicians to review the cases in detail. If the physicians required more tests for decision making, the results of all required tests were given and were also recorded. Physicians were also asked to complete the "Physician Questionnaire" that included questions about their institutions, number of patients they examine in routine daily practice, years of working as a clinician, whether PFT and chest X-rays were performed in their institution, his/her routine procedures to examine a new COPD patient, and whether he/she routinely used a questionnaire to assess the symptoms and/or health-related quality of life of patients. All questionnaires were collected by the interviewers and transferred to the study center for data management.

\section{Statistical analyses}

Predictive Analytics Software for Windows Version 18.0 (SPSS Inc., Chicago, IL, USA) was used for statistical analysis. In descriptive statistics, categorical variables were expressed as numbers and percentages and numerical variables were expressed as median (minimum-maximum). Two-group comparisons between categorical variables were performed using the Chi-square test or the Fisher's exact test, as required. The Spearman's rho test statistics was used in correlations for non-normal distributed numerical data. Multivariate logistic regression analysis was performed to determine the factors affecting the guideline-concordant diagnosis. Statistical significance level was set at a $P$-value of $<0.05$.

\section{Results}

Of the 50 physicians included in the study, 31 (62\%) physicians were working in a tertiary health care center and the remaining 19 physicians were working in a secondary health care center. The median number of years worked as a specialist was 7 years (range, $1-33$ years), and $65.3 \%$ of the physicians had been working as a specialist for $\leq 10$ years. The median number of patients examined daily by the physicians was 40 (range, 8-90). Spirometry was available in all institutions. Features of the physicians are summarized in Table 2.

While $44 \%(n=22)$ of the physicians performed guidelineconcordant diagnosis in the five COPD cases, only $6 \%$ $(n=3)$ of the physicians performed guideline-concordant diagnosis in all seven cases (Table 3 ). There was a negative correlation between the workload ( $\geq 40$ patient examinations per day) of the physicians and the likelihood of making a
Table 2 Features of the physicians

\begin{tabular}{ll}
\hline Features & \\
\hline Gender & $34(68.0)$ \\
Female & $16(32.0)$ \\
Male & $7.0(1.0-33.0)$ \\
Number of years working as a specialist & $32(65.3)$ \\
Number of physicians working as a specialist for \\
$\quad \leq 10$ years & $17(34.7)$ \\
$>10$ years & $31(62.0)$ \\
Working place & $19(38.0)$ \\
$\quad$ Tertiary health care center & $40.0(8.0-90.0)$ \\
$\quad$ Secondary health care center & $25(52.1)$ \\
Number of patients examined daily & $23(47.9)$ \\
Workload of physicians & $50(100.0)$ \\
$\quad \geq 40$ patient examinations per day & <40 patient examinations per day \\
Performing PFT in the institutions &
\end{tabular}

Note: Data are presented as $n(\%)$ or median (minimum-maximum), where appropriate. Abbreviation: PFT, pulmonary function test.

guideline-concordant diagnosis $(P=0.038$, rho $=-0.417)$. There was no relationship between a guideline-concordant diagnosis and the working place (tertiary or secondary health care center) as well as the number of years worked as a specialist ( $P>0.05$, for both). In multivariate logistic regression analysis performed to determine the factors (including gender, number of years working as a specialist, working place [secondary or tertiary health care center], and number of patients examined daily) affecting the guidelineconcordant diagnosis, working in a tertiary health care center was found to be the only significant factor in favor of establishing a guideline-concordant diagnosis of COPD ( $P=0.029, \mathrm{OR}=6.139$ [95\% CI: $1.20-31.32])$.

Independently from failures in the diagnosis and categorization of COPD patients, guideline-concordant treatment rates were $20 \%, 46 \%, 84 \%, 20 \%, 18 \%$, and $24 \%$ in Cases GOLD A-smoker, GOLD A-nonsmoker, GOLD D-smoker, GOLD B-exsmoker, GOLD B-smoker, and ACOS, respectively. The guideline-concordant treatment rates for guideline-concordant diagnosed and categorized patients were $4 \%, 6 \%, 4 \%, 2 \%$, and $2 \%$ in Cases GOLD A-smoker, GOLD A-nonsmoker, GOLD D-smoker, GOLD B-exsmoker, GOLD B-smoker, and ACOS, respectively. While the lowest rates for guideline-concordant diagnosis and guideline-concordant treatment were seen in Case GOLD A-smoker and Case ACOS, Case GOLD D-smoker was associated with the highest rates of correct answers (Table 3) (details are presented in Table S2).

The rate of guideline-concordant diagnosis among physicians who used appropriate PFT was $38 \%$ in Case 


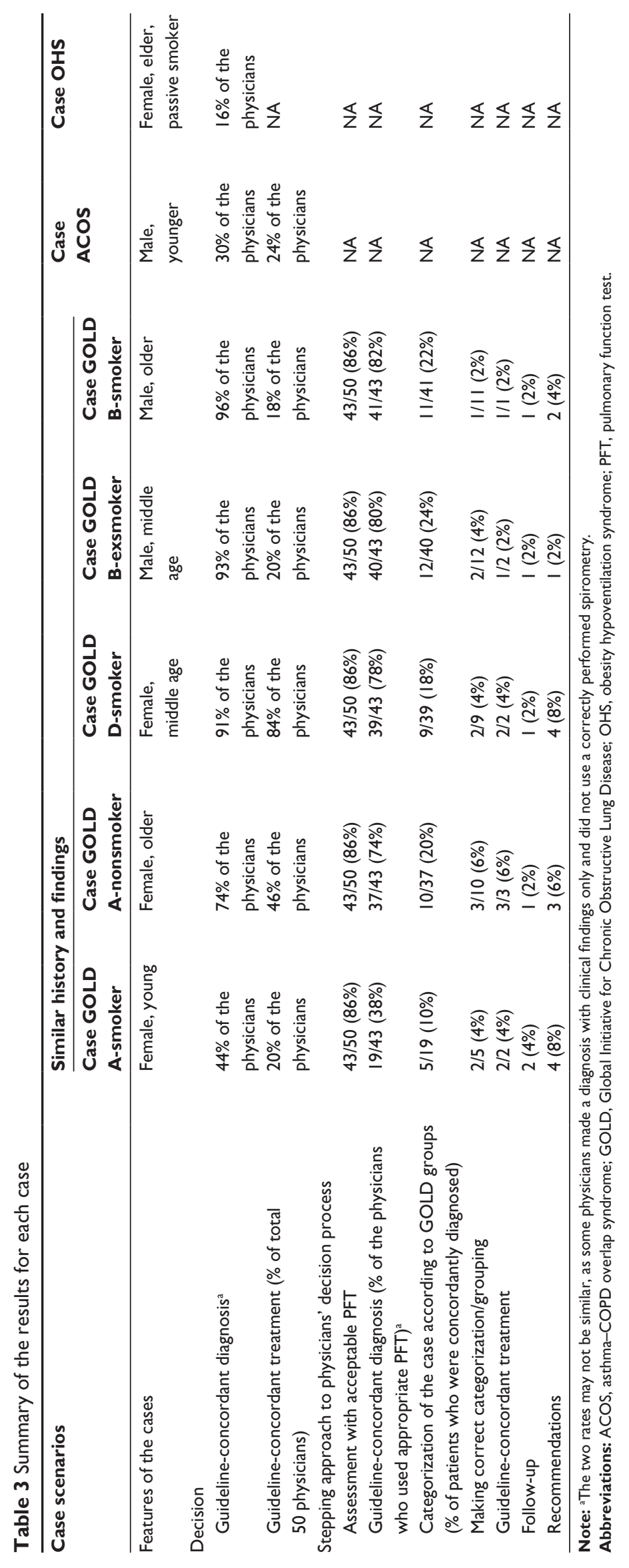




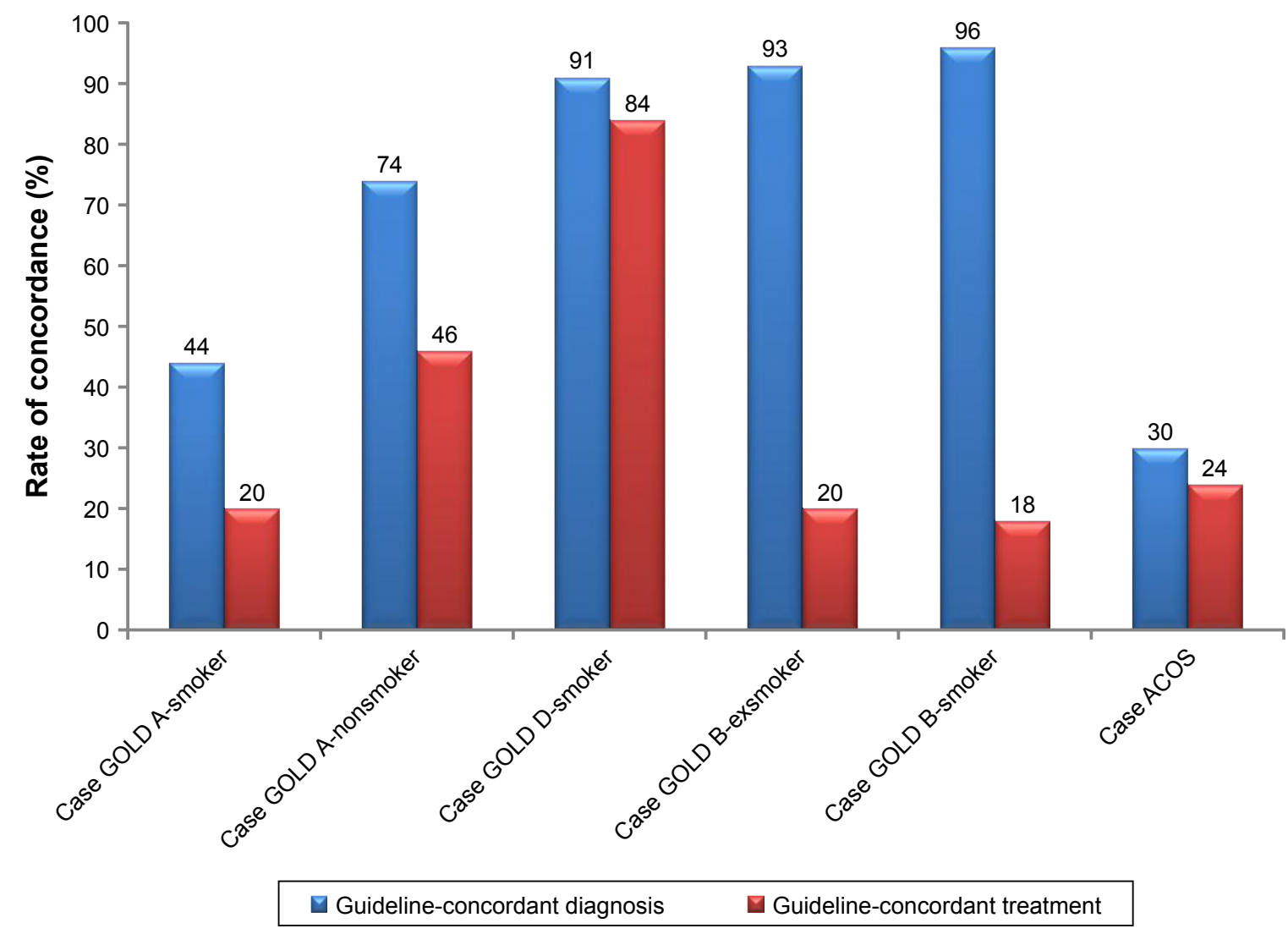

Figure I Rate of guideline-concordant diagnosis and treatment decisions of the physicians according to case scenarios. Abbreviations: ACOS, asthma-COPD overlap syndrome; GOLD, Global Initiative for Chronic Obstructive Lung Disease.

GOLD A-smoker, whereas the rates increased to $74 \%, 78 \%$, $80 \%$, and $82 \%$ in Case GOLD A-nonsmoker, Case GOLD D-smoker, Case GOLD B-exsmoker, and Case GOLD B-smoker, respectively. However, even when a guidelineconcordant diagnosis of COPD was made, only a minority of the physicians (10-24\%) used the GOLD classification and gave guideline-concordant treatment (Figure 1).

\section{Discussion}

The present study evaluated the physicians' behaviors on COPD management using fictitious case scenarios and showed that the patients' characteristics influenced the physicians' decision making. This influence was more apparent in newly diagnosed patients. The classification of the cases according to the GOLD 2015 criteria was also problematic. Besides, correct parameters were used by $10 \%-24 \%$ of the physicians and correct classification was performed only by $2 \%-6 \%$. Moreover, only 4-7 of the physicians used the correct tools for decision making. In spite of the problems in the classification, 18\%-84\% of the physicians prescribed the appropriate treatment.

Although Case GOLD A-smoker and Case GOLD A-nonsmoker were newly diagnosed patients, they differed in age and smoking status, which might have led to different rates in guideline-concordant diagnosis (38\% vs $74 \%$ ). In contrast, Case ACOS was concordantly diagnosed by a higher proportion of the physicians. Case OHS was previously misdiagnosed and treated as COPD and was concordantly diagnosed only by eight physicians. Thus, a previous diagnosis of COPD appears to mislead the physicians to continue with the same mistake. Each new encounter should give the physicians a chance to re-evaluate their patients.

The distribution of COPD patients among the GOLD categories has been evaluated by several studies. The proportion of cases in GOLD A category is roughly between 30\% and $40 \% .^{7-9}$ The national ALPHABET study reported that GOLD A and D were the two most prevalent COPD categories, comprising $41.1 \%$ and $25.0 \%$ of the patients regularly seen at secondary and tertiary health care centers. ${ }^{3}$ As these were the most common categories, physicians are expected to diagnose and manage such patients appropriately in accordance with the GOLD guidelines. However, the rates of guidelineconcordant diagnosis and treatment were low, possibly due to the tendency of physicians to make decisions based on their clinical experience, particularly in these low-risk groups 
with lesser symptoms. ${ }^{3}$ In a multicenter study conducted on 4,094 patients in Italy to investigate whether pulmonologists follow the GOLD guidelines in prescribing COPD treatment, they reported that $62.1 \%$ of the patients were receiving inappropriate treatment in accordance with the GOLD recommendations. ${ }^{10}$ In a study conducted to investigate the rates of adherence to GOLD 2010 guidelines for COPD treatment among pulmonologists in Turkey, overall adherence to GOLD recommendations was $59.5 \% .{ }^{11}$ In another study from Turkey, adherence to guideline recommendations for COPD patients was found to be $48.2 \% .{ }^{12}$ In the present study as well, the rate of guideline-concordant diagnosis among physicians who used appropriate PFT was 38\% in Case GOLD A-smoker, while the rates increased to $74 \%, 78 \%, 80 \%$, and $82 \%$ in Case GOLD A-nonsmoker, Case GOLD D-smoker, Case GOLD B-exsmoker, and Case GOLD B-smoker, respectively. However, even if the guideline-concordant diagnosis of COPD was performed, only a minority $(10 \%-24 \%)$ of the physicians used the GOLD classification.

The diagnosis of COPD should be based on the evaluation of PFTs; however, several previous studies which evaluated the practice patterns of physicians to diagnose COPD have shown that clinical experience has a significant impact on diagnosis and frequently replaces spirometry. An Italian study reported that for patients examined by general practitioners, the COPD diagnosis was based on the physicians' decisions in $30 \% .{ }^{13}$ Similarly, another study from Tasmania reported that $30 \%$ of the patients diagnosed with COPD had no spirometric evaluation. ${ }^{14}$ Moreover, a Chinese study reported that $64.3 \%$ of the hospitals lacked spirometers for COPD diagnosis. ${ }^{15}$ A more recent study from China also reported that knowledge about COPD was fairly low among general practitioners. ${ }^{16}$ In a multinational study including centers from the Europe and the United States, $24 \%$ of the COPD patients reported that they had not undergone a spirometry test. ${ }^{17}$ As can be seen from different population examples, PFTs are not always incorporated in the diagnosis and management of COPD in several parts of the world. The present study showed that spirometry was frequently overlooked in clinical practice in Turkey and that the physicians had problems in interpreting the results when it was performed.

It thus appears that despite educational efforts including scientific meetings, webinars, and national guidelines, important issues remain in the diagnosis and guideline-concordant management of COPD patients in Turkey. There is a strong need to re-evaluate the effectiveness of the current educational tools, to assess clinicians' needs, and to focus more on improving the practice and clinical behavior of pulmonologists caring for COPD patients.

Another interesting finding was the relationship between workload and guideline-concordant diagnosis. The median number of patients examined by the physicians was found to be 40 per day, and with a rough calculation, the physicians had only 12 minutes per patient for examination and decision making. The workload was particularly higher in state hospitals. A recent study by Musellim et al, ${ }^{18}$ however, suggested that in pulmonary practice, the average time allocated to each patient should be 20 minutes and that physicians should spare 25 minutes for patients with chronic lung disorders. In reality, however, under time constraints for each patient, physicians appear to depend more heavily on their clinical experience and possibly use preconceived templates in the evaluation and management of COPD patients. This lack of objective and individual assessment may be another explanation for the low rates of guideline-concordant diagnosis.

One of the limitations of the present study was the use of fictitious cases, which might not reflect real-life practices. However, in order to better evaluate the practice patterns, standard questions on standard clinical problems had to be directed to the physicians. Besides, the findings may be an underestimation of the problem, because the physicians would be less likely to make objective and individualized assessments of patients and be more prone to act on their reflexes due to serious time constraints and several distracting factors in the real-life setting. Another limitation could be the relatively low number of participants, which may raise concerns regarding the generalizability of the findings to the Turkish pulmonologist population. However, each participating physician was from a different medical center and each center was chosen from different provinces according to the EuroStat NUTS 2 regions and, thus, the present study population should be a representative sample.

\section{Conclusion}

To the best of our knowledge, this is the first study that evaluated the physicians' behaviors in the process of diagnosis, treatment, and follow-up of COPD. The results of the present study revealed that the management of COPD patients in Turkey was not in accordance with the GOLD recommendations and was rather based on the physicians' clinical experience. This appeared to be related to heavy workload. Therefore, medical associations and professional societies should put more efforts for advocacy to decrease patient burden on physicians and to improve the quality of patient care. Besides, more work needs to be done both to 
raise awareness of physicians on evidence-based management of COPD patients and to reflect this knowledge in their clinical practice. There is a need for further studies evaluating the effects of educational interventions on physicians' knowledge and behavior.

\section{Acknowledgments}

We thank Clinical Research Manager Clinical Research Organization who provided editorial support and performed statistical analysis funded by Novartis Pharmaceuticals, Turkey.

\section{Author contributions}

All authors contributed toward data analysis, drafting and critically revising the paper, gave final approval of the version to be published, and agree to be accountable for all aspects of the work.

\section{Disclosure}

AK is an employee of Novartis Pharmaceuticals, Turkey. The authors report no other conflicts of interest in this work.

\section{References}

1. Vestbo J, Hurd SS, Agustí AG, et al. Global strategy for the diagnosis, management, and prevention of chronic obstructive pulmonary disease: GOLD executive summary. Am J Respir Crit Care Med. 2013;187(4): 347-365.

2. Republic of Turkey Ministry of Health. Ankara: Chronic Diseases and Risk Factors Survey in Turkey. 2013. Available from: http://sbu.saglik. gov.tr/ekutuphane/kitaplar/khrfai.pdf. Accessed October 18, 2017.

3. Gunen H, Yilmaz M, Aktas O, et al. Categorization of COPD patients in Turkey via GOLD 2013 strategy document: ALPHABET study. Int $J$ Chron Obstruct Pulmon Dis. 2015;10:2485-2494.

4. Sharif R, Cuevas CR, Wang Y, Arora M, Sharma G. Guideline adherence in management of stable chronic obstructive pulmonary disease. Respir Med. 2013;107:1046-1052.
5. Foda HD, Brehm A, Goldsteen K, Edelman NH. Inverse relationship between nonadherence to original GOLD treatment guidelines and exacerbations of COPD. Int J Chron Obstruct Pulmon Dis. 2017;12: 209-214.

6. Fukuhara S, Nishimura M, Nordyke RJ, Zaher CA, Peabody JW. Patterns of care for COPD by Japanese physicians. Respirology. 2005; 10:341-348.

7. Han MK, Muellerova H, Curran-Everett D, et al. GOLD 2011 disease severity classification in COPDGene: a prospective cohort study. Lancet Respir Med. 2013;1(1):43-50.

8. Soriano JB, Alfageme I, Almagro P, et al. Distribution and prognostic validity of the new global initiative for chronic obstructive lung disease grading classification. Chest. 2013;143(3):694-702.

9. Casanova C, Marin JM, Martinez-Gonzalez C; for COPD History Assessment in SpaiN (CHAIN) Cohort, et al. New GOLD classification: longitudinal data on group assignment. Respir Res. 2014;15:3.

10. Corrado A, Rossi A. How far is real life from COPD therapy guidelines? An Italian observational study. Respir Med. 2012;106:989-997.

11. Sen E, Guclu SZ, Kibar I, et al. Adherence to GOLD guideline treatment recommendations among pulmonologists in Turkey. Int $J$ Chron Obstruct Pulmon Dis. 2015;10:2657-2663.

12. Turan O, Emre JC, Deniz S, Baysak A, Turan PA, Mirici A. Adherence to current COPD guidelines in Turkey. Expert Opin Pharmacother. 2016; 17:153-158.

13. Caramori G, Bettoncelli G, Tosatto R, et al. Underuse of spirometry by general practitioners for the diagnosis of COPD in Italy. Monaldi Arch Chest Dis. 2005;63(1):6-12.

14. Walters JA, Hansen E, Mudge P, Johns DP, Walters EH, WoodBaker R. Barriers to the use of spirometry in general practice. Aust Fam Physician. 2005;34(3):201-203.

15. Zheng J. A nationwide questionnaire survey on clinical application of pulmonary function testing in China. Zhonghua Jie He He Hu Xi Za Zhi. 2002;25(2):69-73.

16. Li F, Cai Y, Zhu Y, et al. The evaluation of general practitioners' awareness/knowledge and adherence to the GOLD guidelines in a Shanghai suburb. Asia Pac J Public Health. 2015;27(2):N2067-N2078.

17. Menezes AM, Landis SH, Han MK, et al. Continuing to confront COPD international surveys: comparison of patient and physician perceptions about COPD risk and management. Int J Chron Obstruct Pulmon Dis. 2015;10:159-172.

18. Musellim B, Borekci S, Uzan G, et al. What should be the appropriate minimal duration for patient examination and evaluation in pulmonary outpatient clinics? Ann Thorac Med. 2017;12:177-182.
International Journal of COPD

\section{Publish your work in this journal}

The International Journal of COPD is an international, peer-reviewed journal of therapeutics and pharmacology focusing on concise rapid reporting of clinical studies and reviews in COPD. Special focus is given to the pathophysiological processes underlying the disease, intervention programs, patient focused education, and self management protocols.

\section{Dovepress}

This journal is indexed on PubMed Central, MedLine and CAS. The manuscript management system is completely online and includes a very quick and fair peer-review system, which is all easy to use. Visit $\mathrm{http} / / / \mathrm{www}$.dovepress.com/testimonials.php to read real quotes from published authors. 